\title{
Molecular mechanisms of the drought tolerance in common wheat - a transcriptomic approach
}

\author{
Nowak M. ${ }^{1 *}$, Dudziak K. ${ }^{1}$, Börner A. ${ }^{2}$, Sozoniuk M. ${ }^{1}$, Kowalczyk K. ${ }^{1}$ \\ ${ }^{1}$ Institute of Plant Genetics, Breeding and Biotechnology, University of Life Sciences in Lublin, \\ Lublin, Poland \\ ${ }^{2}$ Leibniz Institute of Plant Genetics and Crop Plant Research (IPK), Gatersleben, Germany \\ *e-mail: michal.nowak@up.lublin.pl
}

Water deficits represent a major global abiotic stress that limits plant productivity by inhibiting their growth and development. The defense mechanism against drought stress in plants contains several elements including enzymatic antioxidant system activation to protect cells against toxic reactive oxygen species (ROS) and the accumulation of osmolytes such as proline. Proline is involved in direct ROS scavenging, stabilize proteins and antioxidant enzymes, and promote cellular signaling. The major signaling system activated in response to stress in plants is based on mitogen activated protein kinases (MAPK) cascades. The objective of presented study was to examine wheat responses to short-term drought, as measured by the expression level of genes involved in signal transduction (MAPK3 and MAPK6), the activity of the antioxidant system and the proline biosynthesis in common wheat seedlings. Furthermore, using intervarietal single chromosome substitution lines (ISCSLs), we identified chromosomes associated with the initial response to short-term drought stress in wheat.

The results of our study revealed that the first reaction of the tested wheat lines was characterized by changes in the catalase transcript level. Furthermore, examined stress induced the expression of genes involved in proline biosynthesis and the MAPK6mediated signaling pathway. In the present study, we identified chromosomes associated with the initial wheat response to short-term stress using a set of common wheat ISCSLs of variety 'Janetzkis Probat' (JP) in the genetic background of 'Saratovskaya 29' (S29) with varying drought tolerance. The data indicated that the substitution of chromosomes 3B, 5A, 7B, and 7D had the largest impact on the expression level of all tested genes and could play a critical function in controlling tolerance to water deficits in the wheat genome. 